\title{
Novel method of histopathological analysis after testicular sperm extraction in patients with nonobstructive and obstructive azoospermia
}

\author{
Gianmartin Cito ${ }^{1 *}$, Maria Elisabetta Coccia ${ }^{2 *}$, Rita Picone ${ }^{2}$, Gabriella Nesi ${ }^{3}$, Andrea Cocci ${ }^{1}$, Sara Dabizzi ${ }^{4}$, Giulio Garaffa ${ }^{5}$, \\ Rossella Fucci ${ }^{2}$, Patrizia Falcone ${ }^{2}$, Francesco Bertocci ${ }^{2}$, Raffaella Santi ${ }^{3}$, Luciana Criscuoli ${ }^{2}$, Sergio Serni' ${ }^{1}$, Marco Carini ${ }^{1}$, \\ Alessandro Natali ${ }^{1}$
}

'Department of Urology, ${ }^{2}$ Assisted Reproductive Technology Centre, ${ }^{3}$ Department of Pathology, and ${ }^{4}$ Sexual Medicine and Andrology Unit, Department of Experimental and Clinical Biomedical Sciences, Careggi Hospital, University of Florence, Florence, Italy; ${ }^{5}$ Institute of Urology, University College London Hospitals, London, UK

Objective: To assess whether the "testicular pool" could be used for histological analysis and whether it gave more accurate information than the standard testicular biopsy.

Methods: Between January 2017 and March 2018, this single-center prospective study included 60 azoospermic men undergoing conventional bilateral testicular sperm extraction. Six samples were excised from each testicle and transferred to an embryologist. One additional biopsy was randomly taken from each testis for a histological analysis. After processing, the testicular pool was also sent for a histological analysis, which showed normal spermatogenesis (NS), hypospermatogenesis (HYPO), maturation arrest (MA), Sertoli cell-only syndrome (SCOS), and tubular atrophy (TA).

Results: Twenty of the 60 patients (33.3\%) had obstructive azoospermia (OA), while the remaining 40 (66.6\%) had nonobstructive azoospermia. Their mean age was 40.5 years. All patients with OA had previously undergone unsuccessful testicular fine-needle aspiration. Successful sperm retrieval (SSR) occurred in 93.3\% of patients. Histological analysis of the testicular biopsy revealed NS in 12 patients (20\%), HYPO and TA in 28 patients (46.6\%), MA in eight patients (13.3\%), and SCOS in 12 patients (20\%). The testicular pool analysis showed NS in 12 patients (20\%), HYPO and TA in 44 patients (73.3\%), MA in four patients (6.6\%), and SCOS in no patients. In four patients with MA (6.6\% of the total sample) and 12 patients with SCOS (20\% of the total sample) according to the standard testicular biopsy, the embryologist found SSR with cryopreservation. Overall, in 44 patients (73.3\%), the testicular pool analysis confirmed the histological findings of the standard testicular biopsy. In the 16 cases (26.6\%) with a discrepancy between the single-biopsy histological findings and SSR, the testicular pool analysis confirmed the embryological data on SSR.

Conclusion: The testicular pool proved to be easily analyzable, practical, manageable, and more accurate for predicting sperm retrieval than standard testicular biopsy.

Keywords: Azoospermia; Histology; Male infertility; Sperm retrieval; Testis

Received: Jun 22, 2018 · Revised: Jul 12, 2018 · Accepted: Aug 7, 2018 Corresponding author: Gianmartin Cito

Department of Urology, Careggi Hospital, University of Florence, Largo Brambilla, 3, 50134 Florence, Italy

Tel:+39-0557949203 Fax:+39-0557949046 E-mail: gianmartin.cito@gmail.com

*These authors contributed equally to this study.

This is an Open Access article distributed under the terms of the Creative Commons Attribution Non-Commercial License (http://creativecommons.org/licenses/by-nc/4.0/) which permits unrestricted non-commercial use, distribution, and reproduction in any medium, provided the original work is properly cited.

\section{Introduction}

Azoospermia, which affects around $1 \%$ of men in the Western world [1], is defined as the complete absence of spermatozoa in the ejaculate after the assessment of centrifuged semen on at least two separate occasions (World Health Organization, 2010). Its etiology is unknown in at least $40 \%$ of cases, possibly because many of the genes that regulate spermatogenesis have not yet been identified [2]. 
Thanks to in vitro fertilization techniques such as intracytoplasmic sperm injection (ICSI), it is potentially sufficient to surgically retrieve a single viable sperm cell through testicular sperm extraction (TESE) to give patients the opportunity to father their own biological child $[3,4]$. When evaluating an azoospermic patient, it is important to establish whether the condition is obstructive (obstructive azoospermia [OA]) or nonobstructive (nonobstructive azoospermia [NOA]), as the management of the two conditions can be completely different. In patients with $\mathrm{OA}$, spermatogenesis is usually normal, but sperm cannot join the rest of the ejaculate due to obstruction at the level of the epididymis, vas deferens, or ejaculatory ducts [5]. NOA, which accounts for around $60 \%$ of all cases of azoospermia, is instead characterized by an intrinsic, often idiopathic, testicular impairment that negatively influences spermatogenesis [6]. Surgical sperm retrieval is offered to patients affected by NOA because, although spermatogenesis is generally compromised, focal areas of spermatogenesis may still exist in their testes [7].

When recanalization surgery is not possible or indicated, in patients with $O A$, sperm can be usually easily retrieved with testicular fine needle aspiration (TEFNA), as normal spermatogenesis (NS) is expected to be diffusely present in the testicles. When attempting sperm retrieval in patients affected by NOA, in contrast, surgical TESE is necessary, with the hope of identifying focal areas of NS $[8,9]$. Viable sperm can be used fresh for an ICSI cycle or can be cryopreserved for future use $[10,11]$. Failed TESE can have important negative emotional and financial implications. Parameters such as hormonal levels, testis volume and histology, and genetic profile are currently used to predict the likelihood of success of sperm retrieval in men affected by NOA, and such predictions may help clinicians provide infertile couples with appropriate counseling and treatment [12]. Since the late 1990s, testis histology has been found to be the most reliable predictive factor of successful sperm retrieval (SSR) in NOA patients [13].

The main limitation of testis biopsy is that it is not representative of the whole anatomy, as usually only a single testicular sample harvested from each testis is sent for histopathological evaluation, although the seminiferous tubules of azoospermic patients are often heterogeneous throughout the testicle [14]. However, sending multiple testis specimens for histopathological analysis could have a negative influence on the endocrine function of the testis, because a larger amount of tissue is sampled [15-18].

In order to study the histology of a larger sample, which would be more representative of the histopathology of the entire testis, in addition to the standard histological biopsy, it is possible to analyze the remnants of TESE specimens after spermatozoa have been extracted by the embryologist. This is because during processing of the TESE sample, only the supernatant with free spermatozoa is cryopreserved or utilized for ICSI, while the testicular solid tissue consisting of the residual seminiferous tubules after stretching, centrifugation, and extracting the spermatozoa, which in the current manuscript is defined as the "testicular pool," is usually disposed. The main objective of the current study was to assess the feasibility of using the testicular pool for histological analysis in azoospermic patients undergoing conventional TESE in order to predict the likelihood of sperm retrieval.

\section{Methods}

\section{Study population}

Between January 2017 and March 2018, 60 azoospermic patients referred to the Assisted Reproductive Technology Centre of the University of Florence (Florence, Italy) were prospectively enrolled in the current study. Patients who presented for semen cryopreservation before undergoing oncologic treatment were excluded.

Azoospermic patients were referred to the Department of Urology for further assessment of the cause of azoospermia and for sperm retrieval after the couple had undergone a complete assessment, including a gynecological evaluation of the female partner. The diagnosis of NOA or OA was based on the patient's history, a physical and genital examination, semen analysis, the patient's endocrine profile, and genetic studies. The data analyzed included age; weight and height; smoking and drinking habits; and history of cryptorchidism, mumps orchitis, varicocele, environmental or radiation exposure, prescribed drug use, previous chemotherapy, and surgical procedures. All patients underwent a genital examination and a scrotal Doppler ultrasound scan to detect testicular volume and to exclude the presence of epididymis head or tail dilatation, unilateral or bilateral absence of the vas deferens, and varicocele. A transrectal ultrasound scan was performed to rule out the presence of prostate median cysts and of anomalies of the seminal vesicles suggestive of obstruction of the male genital tract [18]. Genetic studies were performed, including a karyotype, an analysis of microdeletions in the $Y$ chromosome, and an analysis of mutations in the CFTR gene. The hormonal profile was also documented during hospitalization prior to TESE in all patients. Normal levels of serum follicle-stimulating hormone (FSH), luteinizing hormone (LH), and total testosterone (TT) were considered to be $1.5-8.0 \mathrm{IU} / \mathrm{L}, 1.8-12 \mathrm{IU} / \mathrm{L}$, and $2.7-18 \mathrm{ng} / \mathrm{mL}$, respectively. Prolactin levels were considered normal between 3.0 and $18 \mathrm{ng} / \mathrm{mL}$ and thyroid-stimulating hormone (TSH) levels were considered normal between 0.3 and $5.5 \mathrm{mlU} / \mathrm{L}$. For each patient, the quantity and quality of sperm retrieved at the time of TESE was recorded, including mean total sperm motility and the mean number of cryopreserved Bio System straws.

All participants were adequately counseled, signed a detailed consent form prior to surgery, and agreed to have their data anonymously utilized. All procedures were performed in accordance with 
the ethical standards of the Institutional and National Research Committee and with the 1975 Helsinki Declaration.

\section{TESE technique}

All patients underwent conventional multiple bilateral TESE, under local anesthesia and sedation. The testicles were delivered through a small scrotal incision on the median raphe. Testis samples were harvested through three separate transverse tunical incisions in the upper pole, in lower pole, and at the equator of the testis, respectively.
Depending on the size of the testis, two samples measuring at least $3 \times 2 \times 1 \mathrm{~mm}\left(6 \mathrm{~mm}^{3}\right)$ up to $8 \times 5 \times 3 \mathrm{~mm}\left(120 \mathrm{~mm}^{3}\right)$ were sharply excised from each tunical incision. Once harvested, the fragments were immediately delivered to the embryologist. In addition to the biopsies for TESE, one representative sample was randomly taken from each testis and sent to histopathology. The tunica albuginea was subsequently closed with a running Vicryl 4-0 suture.
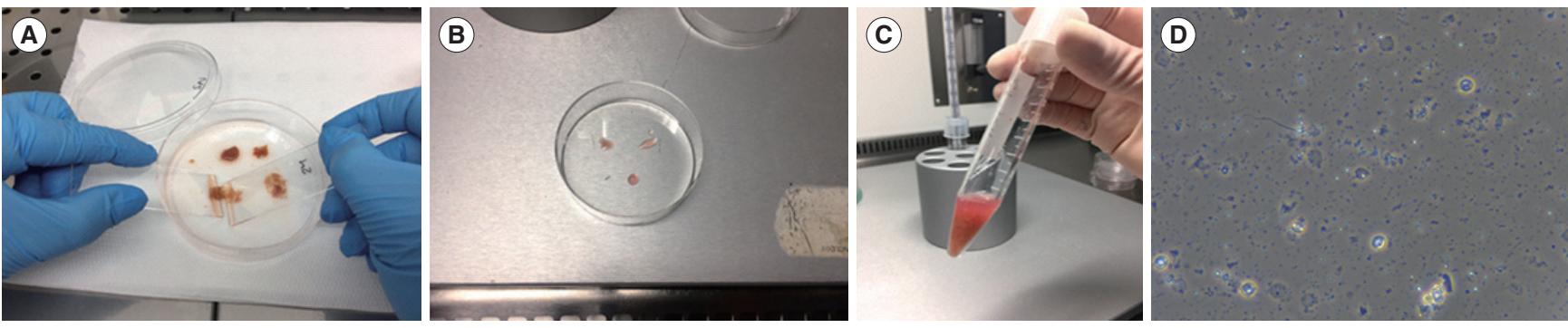

Figure 1. Embryological processing of testicular sperm extraction samples. (A) Stretching of testicular tissue specimens using two sterile glass slides under stereomicroscope. (B) Aliquots of sperm cell suspensions. (C) Testicular pool. (D) Successful sperm retrieval by inverted microscope at $\times 200$.
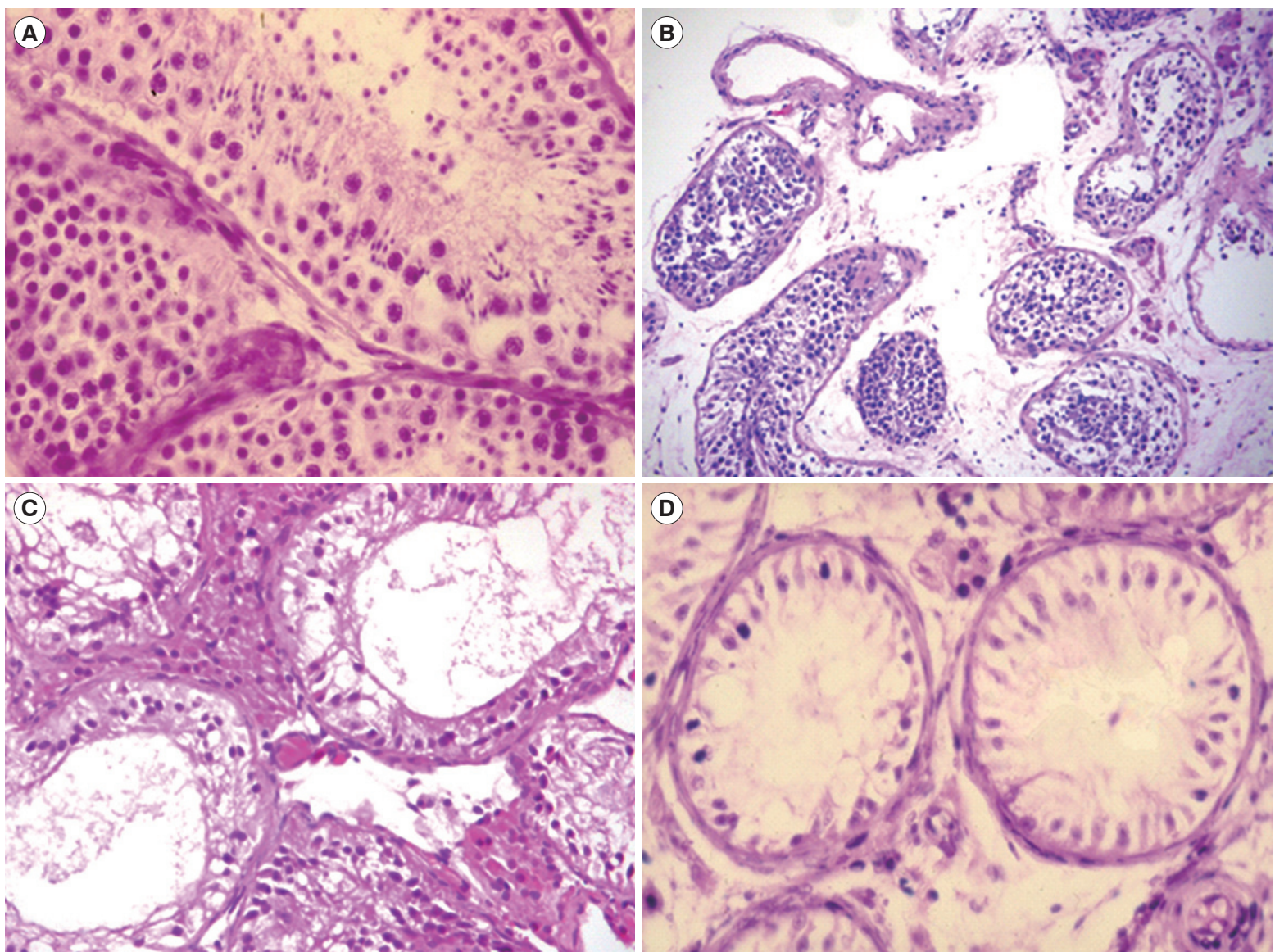

Figure 2. Evaluation of spermatogenesis in the testicular pool using H\&E. Histological patterns of normal spermatogenesis (A), hypospermatogenesis (B), maturation arrest (C), and Sertoli cell-only syndrome (D). Original magnification of all panels, $\times 200$. 


\section{Embryological processing}

The samples of seminiferous tubules were instantaneously examined. Each testicular tissue sample was placed in a sterile Petri dish with a medium solution and then stretched between two sterile glass slides under stereomicroscope. Subsequently, the suspension was directly observed under inverted microscope at $\times 200$ for the first evaluation of the presence of spermatozoa.

Finally, the entire suspension was transferred to tubes and, after sedimentation of the solid tissue, the supernatant containing free sperm cells was processed by centrifugation at 1,800 rpm for $8 \mathrm{~min}$ utes. The pellet was then resuspended in about $300 \mu \mathrm{L}$ of flushing medium. Only the supernatant with free spermatozoa was utilized for assisted reproductive technology purposes.

If retrieved, spermatozoa were either used fresh for an ICSI cycle or alternatively cryopreserved for later use. If the embryologist observed more than 1-2 sperm cells per field (corresponding to an estimated concentration of $0.001 \times 10^{6}$ spermatozoa $/ \mathrm{mL}$ ), sperm retrieval was considered to be successful for use. Usually, if the sperm concentration was sufficient for future utilization, the residual solid tissue was not utilized for cryopreservation. This remnant, defined as the "testicular pool" and consisting of the residual seminiferous tubules, was fixed in $40 \%$ formaldehyde solution and sent for analysis to the histopathology laboratory (Figure 1).

\section{Histological assessment}

A histopathological analysis of the standard biopsy and testicular pool samples was performed by a single dedicated uropathologist. Tissue sections were stained with hematoxylin and eosin. The pathological analysis involved examining at least 100 different sections of seminiferous tubules. The sperm count was carried out under $\times 10$ magnification, exposing several tubules in a single field of vision. In cases of doubt, the presence of spermatozoa was checked with $\times 25$ magnification. Based on the main morphological pattern, the testicular histological findings were categorized as follows (Figure 2): NS, comprising cells at all stages of spermatogenesis and an adequate number of elongated spermatids/spermatozoa; hypospermatogenesis (HYPO), corresponding to a reduction in the number of normal spermatogenetic cells; maturation arrest (MA), characterized by the absence of later stages of spermatogenesis; Sertoli cell-only syndrome (SCOS), when tubules lacked germ cells and were lined with Sertoli cells; and tubular atrophy (TA).

\section{Statistical analysis}

Patient characteristics were described using means and standard deviations for continuous variables and numbers and percentages for dichotomous variables. The Student $t$-test and the Mann-Whitney U-test were used to compare variables, including age and hormone levels. All collected data were evaluated with IBM SPSS ver. 22.0 (IBM Corp., Armonk, NY, USA).

\section{Results}

Data from 60 azoospermic men who underwent sperm retrieval attempts with TESE for fertility treatment at the Assisted Reproductive Technology Centre, University of Florence (Florence, Italy) and whose remnants were sent for histopathological assessment were prospectively collected. Overall, 20 of the 60 participants (33.3\%) had OA and $40(66.6 \%)$ had a clinical presentation of NOA. The baseline characteristics of the study cohort are shown in Table 1. Their mean age at the time of TESE was 40.5 years (range, 33-55 years). The mean age of the female partner was 35.6 years (range, 35-41 years). The participants' mean weight was $79.0 \mathrm{~kg}$ and their mean height was 176.8 $\mathrm{cm}$, with a mean body mass index of $25.26 \mathrm{~kg} / \mathrm{m}^{2}$ (range, $21.91-$ $31.02 \mathrm{~kg} / \mathrm{m}^{2}$ ). Twenty-four patients were smokers (40\%), and 12 (20\%) reported occasional alcohol consumption (usually less than two drinks a week). One patient had previously successfully undergone chemotherapy for the treatment of Hodgkin lymphoma. None

Table 1. Comparison of demographic and clinical parameters between $\mathrm{OA}$ and NOA patients

\begin{tabular}{lccc}
\hline Variable & All patients & OA patient & NOA patient \\
\hline Number & $60(100)$ & $20(33.3)$ & $40(66.6)$ \\
Age $(\mathrm{yr})$ & $40.5 \pm 6.12$ & $38.4 \pm 6.12$ & $41.6 \pm 6.43$ \\
Weight $(\mathrm{kg})$ & $79.0 \pm 11.46$ & $78.4 \pm 10.74$ & $79.3 \pm 10.98$ \\
Height $(\mathrm{cm})$ & $176.8 \pm 5.29$ & $176.6 \pm 5.64$ & $176.4 \pm 4.83$ \\
BMI (kg/m²) & $25.26 \pm 3.37$ & $24.71 \pm 0.06$ & $25.5 \pm 3.65$ \\
Smoking status & $24(40)$ & $4(6.6)$ & $20(33.3)$ \\
Alcohol consumption & $12(20)$ & 0 & $12(20)$ \\
Right TV (mL) & $13.32 \pm 5.48$ & $17.9 \pm 5.59$ & $11.04 \pm 5.48$ \\
Left TV (mL) & $13.18 \pm 6.16$ & $16.0 \pm 5.59$ & $11.78 \pm 6.34$ \\
FSH (IU/L) & $11.56 \pm 5.32$ & $4.68 \pm 5.33$ & $15.0 \pm 5.48$ \\
LH (IU/L) & $4.89 \pm 2.0$ & $3.94 \pm 2.0$ & $5.36 \pm 2.24$ \\
TT (ng/mL) & $4.70 \pm 1.67$ & $4.3 \pm 1.63$ & $4.1 \pm 1.42$ \\
PRL (ng/mL) & $10.1 \pm 6.2$ & $9.8 \pm 5.9$ & $9.5 \pm 5.8$ \\
TSH (mlU/L) & $3.5 \pm 1.2$ & $3.1 \pm 1.2$ & $2.9 \pm 1.4$ \\
Chromosomal anomaly & 0 & 0 & 0 \\
CFTR alteration & $4(6.6)$ & $4(6.6)$ & 0 \\
SSR & $56(93.3)$ & $20(100)$ & $36(90)$ \\
Sperm concentration & $0.12 \pm 0.25$ & $0.24 \pm 0.01$ & $0.05 \pm 0.28$ \\
( $\left.\times 10^{6} / \mathrm{mL}\right)$ & & & \\
Total sperm motility (\%) & $2.13 \pm 0.58$ & $4.8 \pm 0.57$ & $0.8 \pm 0.7$ \\
Straws cryopreserved & $6.8 \pm 3.28$ & $7.2 \pm 3.0$ & $6.6 \pm 3.6$ \\
\hline Values are presented as $n u m b e r$ & \\
\hline
\end{tabular}

Values are presented as number (\%) or mean \pm standard deviation. $\mathrm{OA}$, obstructive azoospermia; NOA, nonobstructive azoospermia; BMl, body mass index; TV, testicular volume; FSH, follicle-stimulating hormone; $\mathrm{LH}$, luteinizing hormone; $\mathrm{TT}$, total testosterone; $\mathrm{PRL}$, prolactin; $\mathrm{TSH}$, thyroid-stimulating hormone; SSR, successful sperm retrieval. 
of the patients suffered from any chronic medical diseases, such as diabetes or cardiovascular disease. Among the 40 patients with NOA, eight (20\%) had a history of cryptorchidism treated with orchidopexy in childhood, and 20 (50\%) had been diagnosed with varicocele, which had been treated with varicocelectomy in four cases. The other NOA patients were diagnosed with idiopathic azoospermia. Among the patients with $\mathrm{OA}$, a CFTR gene abnormality was detected in eight patients: four of them had the more common $\triangle F 508$ mutation and four presented the rare mild mutations $2789+5 \mathrm{G}>\mathrm{A}, 2758+5 \mathrm{G}>\mathrm{A}$, and $2657+5 \mathrm{G}>\mathrm{A}$ present in two patients. All of them had a clinical presentation of congenital bilateral absence of the vas deferens (CBAVD). Four other patients presented CBAVD without CFTR gene anomalies. Three patients had anejaculation due to a previous traumatic spinal cord injury. Five patients had undergone vasectomy in the past. All patients with OA (20/60) had a previous failed TEFNA, without sperm retrieval sufficient for a fresh ICSI cycle, and therefore were offered TESE.

All patients had a normal urine analysis with a negative urine culture, as well as a negative semen culture test. Screening for sexually transmitted diseases was negative in all patients. Scrotal and transrectal ultrasonography documented a median testicular right volume of $13.32 \pm 5.48 \mathrm{~mL}$ (range, $4-25 \mathrm{~mL}$ ) and a median left testicular volume of $13.18 \pm 6.16 \mathrm{~mL}$ (range, $4.3-25 \mathrm{~mL}$ ).

At baseline, the mean serum level of FSH was $11.56 \pm 5.32 \mathrm{IU} / \mathrm{L}$ (range, 2.8-42.7 IU/L), the mean serum level of $\mathrm{LH}$ was $4.89 \pm 2.0 \mathrm{IU} / \mathrm{L}$ (range, $2.7-9.6 \mathrm{IU} / \mathrm{L}$ ), and the mean $\mathrm{TT}$ level was $4.70 \pm 1.67 \mathrm{ng} / \mathrm{mL}$ (range, $1.44-9.5 \mathrm{ng} / \mathrm{mL}$ ). Prolactin and TSH levels were in the normal range in all patients. The overall SSR rate was $93.3 \%$ (56 of 60 patients). Thirty-six of the 40 NOA patients (90\%) had positive sperm retrieval, while SSR occurred in all 20 of the OA patients (100\%). The mean sperm concentration was $0.12 \pm 0.25 \times 10^{6} / \mathrm{mL}$ (range, $0.001-1 \times 10^{6}$ / $\mathrm{mL}$ ). The mean total sperm motility was $2.13 \% \pm 0.58 \%$ (range, $0 \%$ $20 \%$ ). A mean number of $6.8 \pm 3.28$ (range, 1-15). Bio System straws were cryopreserved. The histopathological findings of the testicular pool were compared to those of the standard testicular biopsies.

The histological results of the standard testicular biopsies revealed NS in 12 of 60 patients (20\%), while 28 of 60 patients (46.6\%) had HYPO and TA, eight of 60 patients (13.3\%) had MA, and 12 of 60 patients $(20 \%)$ had SCOS. No cases of germ cell neoplasia in situ were described. The testicular pool showed NS in 12 of 60 patients (20\%), HYPO and TA in 44 of 60 patients (73.3\%), MA in four of 60 patients $(6.6 \%)$, and SCOS in no patients. In four patients with a result of MA $(4 / 60,6.7 \%)$ and 12 patients with a result of SCOS $(12 / 60,20 \%)$ from the standard testicular biopsy histological analysis, the embryologist found positive sperm recovery, allowing subsequent cryopreservation. No testicular pool samples were damaged, and they were therefore adequate for histological assessment. The majority of the testicular
Table 2. Histological patterns depicted by standard testicular histological biopsy and the testicular pool analysis linked to the embryological data of positive sperm retrieval

\begin{tabular}{lcccc}
\hline Method & NS & HYPO-TA & MA & SCOS \\
\hline Standard testicular biopsy & $12(20)$ & $28(46.6)$ & $8(13.3)$ & $12(20)$ \\
Testicular pool & $12(20)$ & $44(73.3)$ & $4(6.6)$ & 0 \\
\hline
\end{tabular}

Values are presented as number (\%).

$\mathrm{NS}$, normal spermatogenesis; $\mathrm{HYPO}$, hypospermatogenesis; TA, tubular atrophy; MA, maturation arrest; SCOS, Sertoli cell-only syndrome.

pool samples consisted of ample amounts of seminiferous tubules with minimal crushing artifacts from the sperm extraction procedure. In 44 of 60 cases (73.3\%), the analysis of the testicular pool confirmed the histopathological features found through the standard testicular biopsy, as verified by the presence or absence of spermatozoa in embryological processing. In the 16 cases (26.7\%) where there was a conflict between the histological results of the standard biopsy and sperm retrieval, the testicular pool analysis proved to be more accurate than the standard histological biopsy as verified by the embryological data. Indeed, in four cases in which the analysis of standard histological biopsy revealed MA and in 12 cases where the standard analysis showed SCOS, the testicular pool demonstrated a histological pattern of HYPO associated with severe TA (Table 2).

\section{Discussion}

The results of the present study show that it is possible to analyze a waste material, such as testicular pool, as a means of predicting SSR. Patients suffering from $O A$ who had previously undergone failed TEFNA were enrolled in the current study, as well as patients with NOA. It should be noted that patients with OA may also have some damage in spermatogenesis, as a result of the long-lasting obstruction causing damage to the spermatogenic tubules [19]. It should also be highlighted that, although patients with NOA have seriously impaired spermatogenesis, they may have some areas showing foci of active NS [20,21]. The patients with OA included in this study previously underwent unsuccessful TEFNA, on the same day of the female oocyte pick-up. Thus, the received aliquots of spermatozoa did not allow a fresh ICSI cycle to be performed, so the oocytes were cryopreserved. The OA patients underwent TESE with multiple incisions because it was necessary to recover as much material as possible for cryopreservation and subsequent utilization with oocytes after thawing. Moreover, in accordance with the literature, we chose to make three incisions - in the upper, lower, and equatorial poles of each testis-to benefit from the focal distribution of spermatogenesis. Nevertheless, in order to minimize testicular damage in OA patients, and in light of their higher likelihood for sperm retrieval than 
NOA patients, we extracted smaller samples from each tunical incision (up to $3 \times 2 \times 1 \mathrm{~mm}$ ).

Testicular histology has previously been suggested to play a role as a predictor of SSR [13,22]. However, testis histology is usually available only after surgical sperm extraction, since diagnostic testicular biopsy is not a cost-effective procedure; therefore, testis histology may mainly be useful for predicting SSR in patients in whom TESE has already failed [11].

In the current series, in four patients (6.7\%) with negative sperm retrieval, the results of the standard histological biopsy and the testicular pool analysis showed the same pattern, equivalent to severe MA of spermatogenesis, and these findings explain the complete absence of mature spermatozoa found by the embryologist. Thus, considering the histological findings, both patients were discouraged from undergoing a further sperm retrieval attempt. Moreover, a single testicular biopsy specimen may not reflect the characteristics of the entire testicular tissue because testicular tubules are usually heterogeneous; therefore, to a certain extent, a single failed attempt at sperm collection cannot exclude the possibility of existing foci of spermatogenesis in the testes $[23,24]$. In this study cohort, in 16 of 60 patients (26.6\%), the embryological evaluation revealed SSR, while the histological results of the standard testicular biopsy showed SCOS or MA. In all those patients, the histological evaluation of the whole testicular pool showed a pattern of HYPO, corresponding to the embryological finding of positive sperm recovery. In all 60 cases $(100 \%)$, the pattern obtained through the testicular pool analysis corresponded to the embryological findings.

The objective of the present study was to identify a way of providing the histopathologist with as much tissue as possible without depleting the testicles of an excessive amount of parenchyma. However, the dimension of the samples chosen was dependent on the baseline characteristics of each patient. Traditionally, only one small sample is used for histology, while the remaining samples are exclusively used for sperm recovery. Depending on the size or number of spermatogenic foci, this may neither be representative of the tissue extracted nor of the whole testis. As the sample for histology is usually obtained simultaneously with the biopsy for sperm retrieval, its usefulness in terms of predicting SSR is limited [25]. Moreover, performing more than a single testicular biopsy for histological purposes cannot be recommended. Of particular note, testosterone levels drop more severely in men with a higher number of biopsies [15-17].

One of the strengths of the current study is that the histological analysis of all testicular samples was performed by the same highlyexperienced pathologist. This is the first study of this new method of histological analysis, in which the remnants of a larger biopsy taken for fertility treatment are analysed in their totality. The principal advantage of this approach is that all available testicular tissue can be used both for sperm retrieval and histopathological evaluation, improving the assessment of spermatogenesis. The main limitations of the current study are the small number of the study cohort and by the fact that the hormone levels were not checked after TESE.

Even if the clinical implementation of the testicular pool analysis may seem unlikely, this technique could prove to be useful, especially in cases where sperm retrieval has failed and the histopathological assessment of the standard testicular biopsy confirms the absence of spermatozoa, but the testicular pool shows the presence of mature spermatozoa (i.e., HYPO). In such cases, the histological evaluation of the testicular pool, as it is more representative of the testis, could guide the clinician to perform the surgical procedure a second time. In our case study, there was no case in which this discrepancy occurred, but we believe that it is an important possibility to highlight. Therefore, although the concept of using remnants for histology represents a very interesting and promising option, its accuracy must be proven in a larger comparative study before it can be implemented in clinical practice. The testicular pool proved to be easily analyzable, practical, manageable, and more accurate than the standard testicular biopsy for predicting sperm retrieval, as it was more representative of the whole testicular parenchyma.

\section{Conflict of interest}

No potential conflict of interest relevant to this article was reported.

\section{References}

1. Donoso P, Tournaye H, Devroey P. Which is the best sperm retrieval technique for non-obstructive azoospermia? A systematic review. Hum Reprod Update 2007;13:539-49.

2. Hamada AJ, Esteves SC, Agarwal A. A comprehensive review of genetics and genetic testing in azoospermia. Clinics (Sao Paulo) 2013;68 Suppl 1:39-60.

3. Verheyen G, Vernaeve V, Van Landuyt L, Tournaye H, Devroey P, Van Steirteghem A. Should diagnostic testicular sperm retrieval followed by cryopreservation for later ICSI be the procedure of choice for all patients with non-obstructive azoospermia? Hum Reprod 2004;19:2822-30.

4. Bukulmez O, Yucel A, Yarali H, Bildirici I, Gurgan T. The origin of spermatozoa does not affect intracytoplasmic sperm injection outcome. Eur J Obstet Gynecol Reprod Biol 2001;94:250-5.

5. Naru T, Sulaiman MN, Kidwai A, Ather MH, Waqar S, Virk S, et al. Intracytoplasmic sperm injection outcome using ejaculated sperm and retrieved sperm in azoospermic men. Urol J 2008;5:106-10.

6. Jarow JP, Espeland MA, Lipshultz LI. Evaluation of the azoospermic patient. J Urol 1989;142:62-5. 
7. Tash JA, Schlegel PN. Histologic effects of testicular sperm extraction on the testicle in men with nonobstructive azoospermia. Urology 2001;57:334-7.

8. Vicari E, Grazioso C, Burrello N, Cannizzaro M, D'Agata R, Calogero AE. Epididymal and testicular sperm retrieval in azoospermic patients and the outcome of intracytoplasmic sperm injection in relation to the etiology of azoospermia. Fertil Steril 2001;75:2156.

9. Esteves SC, Miyaoka R, Orosz JE, Agarwal A. An update on sperm retrieval techniques for azoospermic males. Clinics (Sao Paulo) 2013;68 Suppl 1:99-110.

10. Gil-Salom M, Romero J, Rubio C, Ruiz A, Remohi J, Pellicer A. Intracytoplasmic sperm injection with cryopreserved testicular spermatozoa. Mol Cell Endocrinol 2000;169:15-9.

11. Schroeder-Printzen I, Zumbe J, Bispink L, Palm S, Schneider U, Engelmann $U$, et al. Microsurgical epididymal sperm aspiration: aspirate analysis and straws available after cryopreservation in patients with non-reconstructable obstructive azoospermia. Hum Reprod 2000;15:2531-5.

12. Schlegel PN. Causes of azoospermia and their management. Reprod Fertil Dev 2004;16:561-72.

13. Tournaye H, Verheyen G, Nagy P, Ubaldi F, Goossens A, Silber S, et al. Are there any predictive factors for successful testicular sperm recovery in azoospermic patients? Hum Reprod 1997;12:80-6.

14. van Casteren NJ, Boellaard WP, Dohle GR, Weber RF, Kuizinga MC, Stoop H, et al. Heterogeneous distribution of ITGCNU in an adult testis: consequences for biopsy-based diagnosis. Int J Surg Pathol 2008;16:21-4.

15. Manning M, Junemann KP, Alken P. Decrease in testosterone blood concentrations after testicular sperm extraction for intracytoplasmic sperm injection in azoospermic men. Lancet 1998;352:37.

16. Takada S, Tsujimura A, Ueda T, Matsuoka Y, Takao T, Miyagawa Y, et al. Androgen decline in patients with nonobstructive azo- ospemia after microdissection testicular sperm extraction. Urology 2008;72:114-8.

17. Everaert K, De Croo I, Kerckhaert W, Dekuyper P, Dhont M, Van der Elst J, et al. Long term effects of micro-surgical testicular sperm extraction on androgen status in patients with non obstructive azoospermia. BMC Urol 2006;6:9.

18. Lotti F, Maggi M. Ultrasound of the male genital tract in relation to male reproductive health. Hum Reprod Update 2015;21:56-83.

19. Colpi GM, Pozza D. Diagnosing male infertility: new possibilities and limits. Basel: Karger; 1992.

20. Moein MR, Moein MR, Ghasemzadeh J, Pourmasoumi S. Evaluation of sperm retrieval rate with bilateral testicular sperm extraction in infertile patients with azoospermia. Iran J Reprod Med 2015;13:711-4.

21. Cito G, Coccia ME, Dabizzi S, Morselli S, Della Camera PA, Cocci A, et al. Relevance of testicular histopathology on prediction of sperm retrieval rates in case of non-obstructive and obstructive azoospermia. Urologia 2018;85:60-7.

22. Caroppo E, Colpi EM, Gazzano G, Vaccalluzzo L, Scroppo FI, D’Amato $\mathrm{G}$, et al. Testicular histology may predict the successful sperm retrieval in patients with non-obstructive azoospermia undergoing conventional TESE: a diagnostic accuracy study. J Assist Reprod Genet 2017;34:149-54.

23. Plas E, Riedl CR, Engelhardt PF, Muhlbauer H, Pfluger H. Unilateral or bilateral testicular biopsy in the era of intracytoplasmic sperm injection. J Urol 1999;162:2010-3.

24. Kalsi J, Thum MY, Muneer A, Abdullah H, Minhas S. In the era of micro-dissection sperm retrieval ( $m$-TESE) is an isolated testicular biopsy necessary in the management of men with non-obstructive azoospermia? BJU Int 2012;109:418-24.

25. Verheyen G, Popovic-Todorovic B, Tournaye H. Processing and selection of surgically-retrieved sperm for ICSI: a review. Basic Clin Androl 2017;27:6. 